\title{
Inferior vena cava hypoplasia with right hepatic vein and accessory inferior hepatic vein shunt
}

\author{
N. Çullu ${ }^{1}$, Ö. Yeniçeri², M. Deveer ${ }^{1}$, H. Tetiker ${ }^{3}$ \\ ${ }^{1}$ Department of Radiology, Muğla Sıtkı Koçman University, Faculty of Medicine, Muğla, Turkey \\ 2Department of Radiology, Yücelen Hospital, Muğla, Turkey \\ ${ }^{3}$ Department of Anatomy, Muğla Sıtkı Koçman University, Faculty of Medicine, Muğla, Turkey
}

[Received 19 June 2013; Accepted 31 July 2013]

\begin{abstract}
Inferior vena cava (IVC) hypoplasia is a rare condition. Venous blood flow is usually provided through collaterals in the azygos or hemiazygos venous systems. However, portosystemic shunts with intrahepatic venous collateral are extremely rare. The case is presented here of a large shunt between the right hepatic vein, accessory inferior hepatic vein, and inferior vena cava in a 37-year-old female patient with IVC hypoplasia. (Folia Morphol 2014; 73, 1: 99-101)
\end{abstract}

Key words: inferior vena cava, hypoplasia, ultrasonography, magnetic resonance imaging

\section{INTRODUCTION}

Inferior vena cava (IVC) hypoplasia is an uncommon condition, and may be asymptomatic. Venous blood flow is usually provided through collaterals in the azygos or hemiazygos venous systems. Portosystemic shunts with intrahepatic venous collateral are extremely rare. The case is presented here of a large shunt between the right hepatic vein, accessory inferior hepatic vein, and vena cava inferior in a 37-year-old female patient with IVC hypoplasia.

\section{CASE REPORT}

A 37-year-old female presented at the internal medicine department for a check-up examination without any specific complaint. Clinical examination and laboratory tests were normal. In the ultrasonography (USG) examination, a large hepatic vein was detected. An abnormal vascular structure of $15 \mathrm{~mm}$ diameter in the right lobe of the liver was observed on the abdominal USG. Colour and pulse Doppler USG demonstrated a hepatopetal flow within the abnormal vascular structure. The portal venous system was evident and in a normal configuration. The retrohepatic IVC was not identified. The upper end of the IVC finished in the retro hepatic region where it entered through the liver and made large anastomotic channels with the right hepatic vein in the peripheral regions of the right lobe of the liver (Fig. 1). Then, the patient was examined by magnetic resonance imaging (MRI), which determined (on FIESTA sequence) advanced narrowing of a $3 \mathrm{~cm}$ section in the retrohepatic IVC. In addition, there were widely dilated shunts between the accessory inferior hepatic vein and the right hepatic vein. Aneurismatic widening was observed at the level of the accessory inferior hepatic vein origin (Fig. 2). The blood in the distal of hypoplastic segment of the IVC drained to the proximal segment through the shunts between the accessory inferior hepatic vein and the right hepatic vein (Fig. 3). The liver function tests and other laboratory test results were normal. This patient, who has no clinical findings, has been under observation for years without any treatment.

\section{DISCUSSION}

The embryological development of the IVC is quite a complicated process. The IVC develops from sequentially anastomosis and regression of 3 paired venous channels [7]. The sixth and seventh weeks of gestation are the most critical period for this and

Address for correspondence: Dr N. Çullu, Assist. Prof., Muğla Sıtkı Koçman School of Medicine, Department of Radiology, Central Campus, 48000, Muğla, Turkey, tel: (+90252) 21110 00, fax: (+90252) 22392 80, e-mail: nesatcullu77@gmail.com 


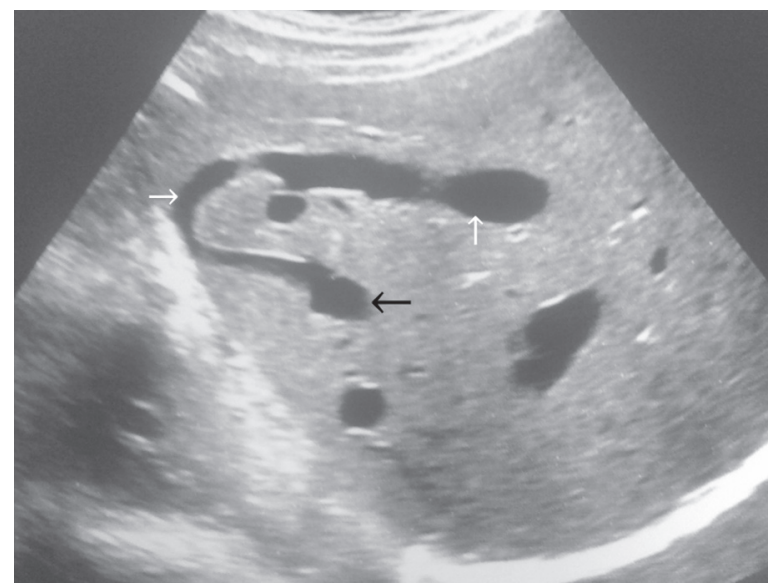

Figure 1. The accessory inferior hepatic vein (black arrow) and right hepatic vein (vertical white arrow) found between the shunt (horizontal white arrow) in the ultrasonography examination of the 37-year-old female.
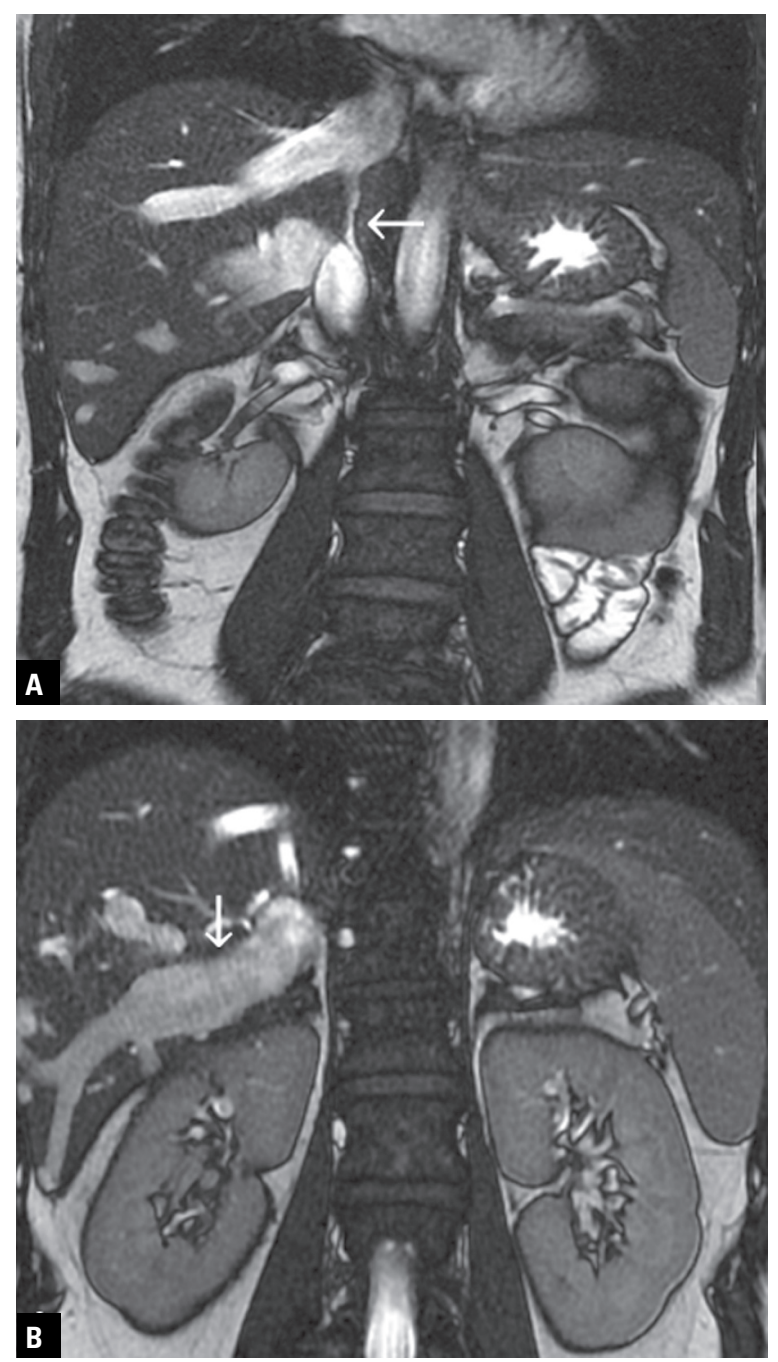

Figure 2. Hypoplasia of the retrohepatic inferior vena cava (white arrow) $(\mathbf{A})$ and dilated accessory inferior hepatic vein (white arrow) (B).

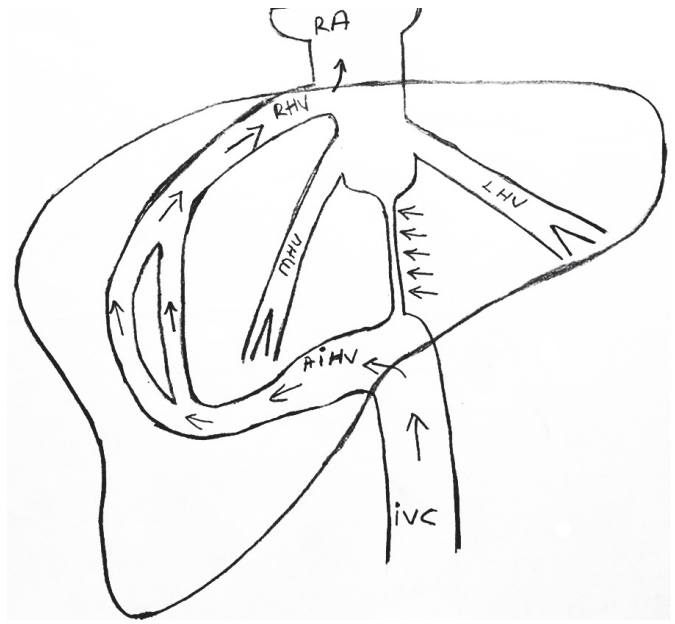

Figure 3. Schematic representation of the inferior vena cava hypoplasia (IVC) (arrows) with right hepatic vein (RHV) and accessory inferior hepatic vein (AIHV) shunt; MHV — middle hepatic vein; LHV — left hepatic vein; RA — right atrium.

until the eighth week, the differentiation of cava at cranial levels continues almost as in adulthood. Hypoplasia or agenesis of retrohepatic vena cava segment and drainage over hepatic veins are the results of abnormal regression or organisation in the early gestational period. Most researchers believe that the vast majority of cases of IVC interruption are due to congenital anomalies $[3,8]$, either a lack of development of venous channels or lack of persistence of anastomotic channels.

IVC anomalies with agenetic segment are very rare. Alternative routes of venous return in IVC interruption are the lumbar vertebral pathways, the anterior abdominal wall veins and the transumblical portocaval way $[4,5]$. The lumbar vertebral pathways can be divided into central and intermediate channels. The central channels (azygos vein etc.) are the most frequent and the first opened pathways [4]. Hemiazygos continuation is reported less frequently than azygos continuation [1]. This drainage to the portal system and hepatic veins is very rarely reported. These cases are generally asymptomatic, although in some cases, symptoms can appear, such as right upper quadrant pain. Our patient was asymptomatic. colour Doppler examinations added to USG demonstrated a hepatopetal flow and warning about possible IVC pathology. MRI gives better visualisation of both the hypoplastic area and the shunt and afferent and efferent vessels. In the current case, the blood in the distal hypoplastic segment of the IVC drained to the proximal segment through the shunts between the accessory inferior hepatic vein and the right 
hepatic vein. Magnetic resonance and multidetector computed tomography venography imaging could be beneficial for the demonstration of shunt and delineation of collateral circulation $[2,6]$.

\section{CONCLUSIONS}

In conclusion, MRI examination is needed to demonstrate in detail congenital anomalies of IVC and establish proper diagnosis.

\section{REFERENCES}

1. Anderson A, Adams P, Burke B (1961) Anomalous inferior vena cava with azygos continuation. J Pediatr, 59: 370-383.

2. Bayraktutan U, Kantarci M, Olgun H, Kizrak Y, Pirimoglu B (2012) Partial anomalous pulmonary venous return associated with vascular anomalies of the aorta: multidetector computed tomography findings. Folia Morphol, 2: 115-117.
3. Debbing E, Tielemans Y, Jolie E, Van den Brande P (1993) Congenital absence of inferior vena cava. Eur J Vasc Surg, 7: 201-203.

4. Ferris E, Vittimberga FJ, Byrne JJ, Nabseth DC, Shapiro JH (1967) The inferior vena cava after ligation and plication. Radiology, 89: 1-10.

5. Gorenstein A, Gordon RL, Abu-Dalu K (1981) Collateral pathways in inferior vena cava obstruction in children, including the portocaval route. Pediatr Radiol, 10: 225-228.

6. Patrick C, Emmanuel PP, Pierre-Yves M, Fabiani P, Dıaıne B, Padovani B (1999) Inferior vena cava hypoplasia with intrahepatic venous continuation: sonographic, angiographic and MR feature including MR angiography. Clin Imag, 23: 99-102.

7. Rosse C, Gaddum-Rosse P (1999) Hollinshead's textbook of anatomy. Lippincott Williams \& Wilkins, Philadelphia.

8. Sandercoe GD, Brooke-Cowden GL (2003) Developmental anomaly of inferior vena cava. ANZ J Surg, 73: 356-360. 